\title{
BMJ Open Variation in the practice of discontinuing mechanical ventilation in critically ill adults: study protocol for an international prospective observational study
}

\author{
Karen E A Burns, ${ }^{1,2,3}$ Leena Rizvi, ${ }^{2}$ Deborah J Cook, ${ }^{4,5}$ Peter Dodek, ${ }^{6}$ \\ Arthur S Slutsky, ${ }^{1,2,3}$ Andrew Jones, ${ }^{7}$ Jesus Villar, ${ }^{8,9}$ Farhad N Kapadia, ${ }^{10}$ \\ David J Gattas, ${ }^{11,12}$ Scott K Epstein, ${ }^{13}$ Maureen O Meade, ${ }^{5}$ On behalf of Canadian \\ Critical Care Trials Group
}

To cite: Burns KEA, Rizvi L, Cook DJ, et al. Variation in the practice of discontinuing mechanical ventilation in critically ill adults: study protocol for an international prospective observational study. BMJ Open 2019;9:e031775. doi:10.1136/ bmjopen-2019-031775

- Prepublication history and additional material for this paper are available online. To view these files, please visit the journal online (http://dx.doi. org/10.1136/bmjopen-2019031775).

Received 22 May 2019 Revised 29 July 2019 Accepted 30 July 2019

Check for updates

(c) Author(s) (or their employer(s)) 2019. Re-use permitted under CC BY-NC. No commercial re-use. See rights and permissions. Published by BMJ.

For numbered affiliations see end of article.

Correspondence to Dr Karen E A Burns; BurnsK@smh.ca

\section{ABSTRACT}

Introduction Research supports the use of specific strategies to discontinue mechanical ventilation (MV) in critically ill patients. Little is known about how clinicians actually wean and discontinue MV in practice or the association between different discontinuation strategies and outcomes. The primary objective of this study is to describe international practices in the use of (1) daily screening for readiness to discontinue MV, (2) modes of MV used before initial discontinuation attempts, (3) weaning and spontaneous breathing trial (SBT) protocols, (4) SBT techniques and (5) sedation and mobilisation practices to facilitate weaning and discontinuation. The secondary objectives are to identify patient characteristics and time-dependent factors associated with use of selected strategies, investigate associations between SBT outcome (failure vs success) and outcomes, explore differences between patients who undergo an SBT early versus later in their intensive care unit (ICU) stay, and investigate the associations between different SBT techniques and humidification strategies on outcomes.

Methods and analysis We will conduct an international, prospective, observational study of MV discontinuation practices among critically ill adults who receive invasive MV for at least 24 hours at approximately 150 ICUs in six geographic regions (Canada, USA, UK, Europe, India and Australia/New Zealand). Research personnel at participating ICUs will collect demographic data, data to characterise the initial strategy or event that facilitated discontinuation of MV (direct extubation, direct tracheostomy, initial successful SBT, initial failed SBT or death before any attempt could be made), clinical outcomes and site information. We aim to collect data on at least 10 non-death discontinuation events in each ICU (at least 1500 non-death discontinuation events).

Ethics and dissemination This study received Research Ethics Approval from St. Michael's Hospital (11-024) Research ethics approval will be sought from all participating sites. The results will be disseminated through publications in peer-reviewed journals. Trial registration number NCT03955874.
Strengths and limitations of this study

- This study will characterise variation in practice related to mechanical ventilation discontinuation internationally and associations between the use of different discontinuation strategies and clinically important outcomes.

- We aimed to recruit a similar number of participating intensive care units (ICUs) in each region recognising that participation of a larger number of ICUs in any one region could result in greater observed practice variation and conversely, inclusion of only a few ICUs in any one region may underestimate true weaning practice variation.

- A study of this scope and rigour focused on mechanical ventilation discontinuation in both academic and community ICUs, involving individual site-training, prospective and consecutive patient inclusion, event identification and data collection, and patient-level data validation has not been conducted previously.

- Challenges will include the need to obtain prospective consent by a small number of research ethics boards, variation in time to ethics and contract approvals, and the number of ICUs that we will need to recruit to achieve our target number of participating ICUs.

\section{INTRODUCTION}

Mechanical ventilation (MV) is the prototypical life support technology used in the intensive care unit (ICU). Most adult patients who are admitted to ICUs require either invasive or noninvasive MV for a proportion of their stay. A key component of MV support is the process of weaning or discontinuing MV. During MV discontinuation, the work of breathing is transferred from the ventilator back to the patient and ventilator support is either abruptly or gradually withdrawn. 
Approximately $40 \%$ of the total duration of invasive MV is spent in the weaning phase. ${ }^{12}$ Prolonged invasive MV is responsible for a large portion of ICU costs and is associated with the development of complications including ventilator-associated pneumonia, ${ }^{3}$ sinusitis $^{4}$ and respiratory muscle weakness. ${ }^{3}$ Thus, minimising the duration of MV has been identified as a priority for improvement in the field of critical care. ${ }^{5}$

Randomised controlled trials, meta-analyses and clinical practice guidelines support the use of specific strategies during weaning including the early identification of weaning candidates using weaning protocols, ${ }^{6-9}$ tests to determine the patient's ability to breathe spontaneously (or spontaneous breathing trials (SBTs)), ${ }^{10-13}$ and selected modes of ventilation or strategies (pressure support (PS) and SBTs (using PS or T-piece). ${ }^{14-16}$ However, little is known about how clinicians actually discontinue MV (direct extubation (no prior SBT), initial SBT, direct tracheostomy) in practice or about the impact of different discontinuation practices on clinical outcomes. ${ }^{17}$

Weaning is a collaborative effort involving the skills of diverse healthcare providers including respiratory therapists (RTs), critical care nurses, physiotherapists, kinesiotherapists, dietitians, pharmacists and physicians. Availability of personnel and organisational factors (teaching affiliation, funding status (public vs private), organisational models (open vs closed) and ICU processes of care) may influence how evidence is adopted into clinical practice and affect outcomes. ${ }^{5} 1819$ Despite strong recommendations for the use of weaning protocols, several factors may limit their use in practice including the need for multidisciplinary education, scale of implementation and strategies to ensure ongoing compliance. $^{20}$

In this International, Prospective, Observational Study of Mechanical Ventilation Discontinuation Practices (hereafter referred to as the IOS study), we aim to describe variation in practice variation in discontinuing MV at national and international levels, describe the impact of different practices in discontinuing MV on clinical outcomes and identify important predictors of initial SBT outcome (see the Objectives section).

\section{OBJECTIVES}

\section{Primary objective}

The primary objectives of the IOS study are to describe in the use of (1) daily screening for readiness to discontinue MV, (2) modes of MV used before initial attempts to discontinue MV, (3) weaning and SBT protocols, (4) SBT techniques and (5) sedation and mobilisation practices among geographic regions.

\section{Secondary objectives}

The secondary objectives are to (1) identify patient characteristics and time-dependent factors associated with use of selected discontinuation strategies, (2) investigate associations between SBT outcome (failure vs success) and clinical outcomes (total duration of MV; overall all-cause ICU mortality; overall hospital mortality; proportion of patients off the ventilator and out of the ICU at day 28 after MV initiation; ICU and hospital length of stay (LOS); ICU readmission (during hospitalisation) and reintubation), (3) explore differences between critically ill patients who undergo an SBT early versus later in their ICU stay (eg, defined by the median ICU LOS or time to first SBT) and (4) investigate the associations between different SBT techniques and humidification strategies (heat and moisture exchanger (HME) vs heated humidification $^{21}$ ) on clinical outcomes.

\section{Tertiary objective}

The tertiary objective is to identify important predictors (patient, clinician, SBT, institutional and regional) of initial SBT outcome (success vs failure).

\section{METHODS}

\section{Study population}

We will include all newly admitted critically ill adults who are invasively ventilated for at least 24 hours. We will exclude patients who (1) are transferred to participating ICUs without a documented intubation time, (2) have a tracheotomy/tracheostomy at ICU admission and (3) are already on ventilator settings compatible with an SBT (eg, T-piece, CPAP $\leq 5 \mathrm{cmH}_{2} \mathrm{O}$ or PS $\leq 7 \mathrm{cmH}_{2} \mathrm{O}$ ) at ICU admission. We will exclude patients who reside in participating ICUs for $\geq 24$ hours on the first day of data collection and those readmitted to the index ICU during the study period. We will also exclude patients who are enrolled in interventional studies that mandate explicit weaning protocols.

\section{Study settings}

We will include approximately 150 ICUs in six geographic regions (Canada, USA, UK, Europe, India and Australia/ New Zealand) with similar representation from each region (approximately 25 ICUs). In each ICU, we will collect data on at least 10 non-death discontinuation events and all deaths before any attempt at MV discontinuation can be made. To enhance study feasibility, we will prioritise ICUs that have 15 or more beds.

\section{Study outcomes}

We will classify each new ICU admission over the study period according to the initial strategy that facilitated MV discontinuation into one of five categories: direct extubation, direct tracheostomy, initial SBT success, initial SBT failure or death before any attempt at discontinuation of MV can be made (figure 1). We will describe the association between MV discontinuation practices and clinical outcomes including total duration of MV, ICU mortality, hospital mortality, proportion of patients off the ventilator and out of the ICU at day 28 after MV initiation, ICU and hospital LOS, ICU readmission (during the index hospitalisation) and reintubation within 48 hours of extubation. 


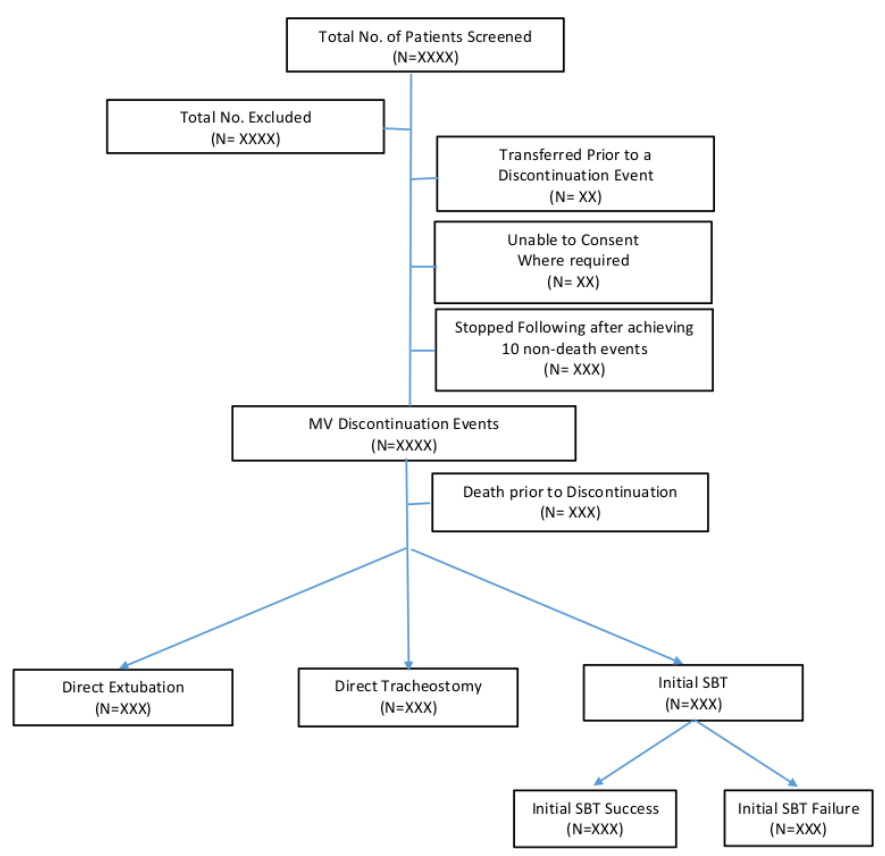

Figure 1 Patient flow. SBT, spontaneous breathing trial MV, mechanical ventilation

\section{DETAILED METHODS}

\section{Phase 1: Study centre identification}

We will use a multimodal approach (information cards, contact with critical care societies and electronic mail correspondence) to identify potential participating centres and ICUs with the goal of collaborating with 25 ICUs in each of the six geographic regions. As in the previously conducted International Weaning Survey of clinicians stated practices in discontinuing $\mathrm{MV},{ }^{17}$ we will define geographic regions based on affiliation with leading regional critical care societies. ${ }^{17}$ To identify potential participating academic and community centres for the IOS study, we enclosed an information card and a separate return envelope with the International Weaning Survey questionnaire.

\section{Phase 2: Data collection forms}

Research staff will prospectively screen the ICU on a daily basis for eligible study patients and record all patients in screening logs (online supplementary appendix 1). After identification of eligible patients, research personnel will follow patients prospectively to ascertain the initial strategy that facilitated MV discontinuation. We will develop web-based data forms to tabulate demographic data (online supplementary appendix 2), data at the time of ICU admission (online supplementary appendix 2), and discontinuation events (direct extubation (online supplementary appendix 3), direct tracheostomy (online supplementary appendix 4), initial successful SBT and up to two subsequent SBTs (online supplementary appendices $5 \mathrm{a}, 5 \mathrm{~b}, 5 \mathrm{c})$, initial failed SBT and up to two subsequent SBTs (online supplementary appendices $5 \mathrm{a}, 5 \mathrm{~b}, 5 \mathrm{c}$ ), or death before any attempt at discontinuation can be made (online supplementary appendix 6). Demographic and ICU admission data will be entered each time a patient meets eligibility criteria. Finally, we will collect information regarding patient outcomes (online supplementary appendix 7) and relevant processes of care (the presence of weaning and SBT protocols, the use of daily screening, and personnel (eg, RTs, nurses, kinesiotherapists or others) involved in weaning) in participating ICUs on separate outcomes (online supplementary appendix 7) and site forms (online supplementary appendix 8), respectively. Research personnel complete paper data collection forms and enter data into the Medidata RAVE System (Medidata Solutions, New York, USA).

\section{Phase 3: Study implementation}

We will prospectively collect data over an approximate 2-week period or until a minimum of 10 non-death discontinuation events are recorded in each ICU. We will permit flexible start dates for variable clinical coverage models (ie, attending weeks of coverage beginning on different days of the week). We will follow patients until successful extubation/disconnection (in patients who have a tracheostomy), death, ICU discharge/transfer or until day 60 for patients who remain dependent on MV. We define the time of successful extubation/disconnection as the time when unsupported spontaneous breathing began (no non-invasive ventilation (NIV) or invasive MV) and was sustained for $>48$ hours after extubation/disconnection. Patients and the public were not involved in study design or implementation.

\section{Phase 4: Data management}

The Applied Health Research Centre of the Keenan Research Centre and the Li Ka Shing Knowledge Institute (St Michael's Hospital, Toronto, Canada) will be the study methods centre. We will use the Medidata RAVE System, a web-based data entry system, to manage study data. We will assign a unique identification code to each participating site based on the region and ICU within the region. The investigative team will transmit queries for missing, incomplete or illogical data to site personnel in the database and by electronic mail. Site personnel will respond to queries and update the database in responses to our queries.

\section{STATISTICAL ANALYSIS}

\section{Sample size estimation}

We will collect data on at least 10 non-death discontinuation events within each participating ICU to obtain at least 1500 non-death discontinuation events. Based on clinical judgement, we anticipate that at least $50 \%$ of discontinuation events will involve an initial SBT (expected range 750-1050 SBTs). Of these, we estimate that $70 \%$ of patients will be successful in an initial SBT, representing 525 initial SBT successes and 225 initial SBT failures. We expect that the rate of accrual of discontinuation events and duration of data collection will differ among the participating ICUs due to the number of ICUs 
beds and patient turnover. We expect to collect sufficient data to identify predictors of initial SBT outcome (failure vs success) using a logistic regression analysis ${ }^{22}{ }^{23}$ with 18 pre-specified variables (online supplementary appendix 9 ), assuming 10 events per degree of freedom. ${ }^{24}$

\section{Primary outcome}

We will use descriptive statistics to summarise variation in weaning practices among geographic regions and clinical outcomes associated with the different discontinuation strategies. For binary outcomes, we will compare differences among the four main discontinuation strategies (direct extubation, direct tracheostomy, initial SBT success and initial SBT failure) using the $\chi^{2}$ test (alternatively, the Fisher's exact test). For continuous outcomes, we will compare means (for normally distributed data) using analysis of variance (ANOVA) or medians (for non-normally distributed data) using the Kruskal-Wallis test or the Mann-Whitney U test, as appropriate. ${ }^{22}$

\section{Secondary and tertiary outcomes}

We will use Cox Proportional Hazards modelling to explore the relationship between patient characteristics and time dependent factors (clinical conditions that developed after enrollment and before initial discontinuation events) associated with the use of selected discontinuation strategies (dependent variable). Among critically ill adults who undergo an initial SBT, we will use descriptive statistics including proportions and means \pm standard deviations (alternatively, medians and IQRs) to summarise binary and continuous variables, respectively. We will use the $\chi^{2}$ test (alternatively, Fisher's exact test) and Student's t-test (alternatively, Mann-Whitney U test) to investigate associations between binary and continuous outcomes, respectively. To investigate the influence of different SBT techniques (eg, CPAP and PS) on clinical outcomes we will use $\chi^{2}$, ANOVA or Mann-Whitney U testing according to the characteristics of the outcome. We will perform a logistic regression analysis to identify potential predictor variables (patient, clinician, SBT, institutional and regional) (online supplementary appendix 9) that are associated with initial SBT outcome. ${ }^{22-24}$ A PhD level biostatistician will conduct all analyses in SAS V.9.1.

\section{ETHICS AND DISSEMINATION}

The IOS study was designed in accordance with ethical principles outlined in the Declaration of Helsinki ${ }^{25}$ and the ethical requirements of participating sites in their respective jurisdictions. We will seek ethics approval and negotiate contracts from all participating sites, where required, before study initiation. The results will be disseminated through publications in peer-reviewed journals.

\section{PATIENT AND PUBLIC INVOLVEMENT}

No patient or public involvement.

\section{DISCUSSION}

The IOS study seeks to quantify the magnitude of practice variation in liberating critically ill adults from invasive MV and associations between use of selected discontinuation strategies and clinical outcomes. Building on previous work including national and international weaning surveys, summarising intensivists' stated practices in weaning critically ill adults from invasive ventilation, the IOS study will examine how clinicians actually discontinue MV in ICUs from six geographic regions of the world. ${ }^{1726}$ A study of this scope and rigour focused on MV discontinuation in both academic and community ICUs, involving individual site-training, prospective and consecutive (where possible) patient inclusion, event identification and data collection, and patient-level data validation (through a process involving individual data review, and detailed query generation and resolution over electronic mail and within Medidata RAVE) has not been conducted previously. We will also identify important predictors (patient, clinician, SBT, institutional and regional) of initial SBT outcome (success vs failure).

We hypothesise that significant practice variation will exist among ICUs in different geographic regions in the use of daily screening, preferred methods of support before discontinuation, use of written directives for weaning and SBT conduct, in the conduct SBTs and in sedation and mobilisation practices. We predict that patients who undergo a tracheostomy or fail an initial SBT will experience higher overall mortality, reintubation, and readmission rates, and longer ICU and hospital LOS. Furthermore, these patients will be more likely to remain in the ICU and on invasive MV at day 28 after MV initiation. We anticipate that patient characteristics (eg, age, patient type (medical vs surgical), illness severity and prior history of heart failure) and time-dependent factors (development of acute respiratory distress syndrome, heart failure, neutropaenia, a bleeding diathesis or the requirement for dialysis) will be associated with the use of selected discontinuation strategies. In addition, we postulate that patients who fail an initial SBT and those who undergo an SBT later in their stay will experience significantly higher mortality, reintubation and readmission rates, longer ICU and hospital LOS, and will be more likely to remain in the ICU and on MV at day 28 compared with patients who pass an initial SBT and those who undergo an SBT earlier in their ICU stay. We do not anticipate finding differences in clinical outcomes based on the SBT technique used; however, we predict that use of HME will result in more initial SBT failures, especially among patients who undergo an SBT later in their ICU stay. Finally, we expect to identify important patient-related (age, patient type, illness severity), clinician-related (presence of RTs, time spent in clinical practice), SBT-related (use of daily screening, SBT technique) and institutional-related predictors (public vs private funding, university affiliation, presence of written directives for weaning and SBT conduct) of SBT outcome. 
We encountered several challenges in designing and coordinating the IOS study. First, in designing the study we recognised that participation of a larger number of ICUs in any one geographic region could result in greater observed practice variation and conversely inclusion of only a few ICUs in any one region may underestimate true weaning practice variation. As such, we aimed to recruit a similar number of participating ICUs in each region. Second, we secured peer-reviewed funding through a Canadian Institutes of Health Research Operating/Industry partnered grant opportunity $(80: 20)$ to provide site investigators with a payment $(\$ \mathrm{C} 750)$ for organising local approvals and prospective data collection. Despite the IOS study being investigator-initiated and peer-review funded, partial industry funding resulted in unanticipated requests for payment from several ethics committees for ethics review. Third, to address variation in ventilator terminology, we used standard terminology, ${ }^{27}$ where feasible and engaged our international collaborators in developing and pilot testing data collection forms. Fourth, to minimise the potential for selection bias introduced by the need to obtain prospective consent, ${ }^{28}$ we intended to implement this purely observational study with a waiver of consent. To ensure that family members were aware that the IOS study was in progress, we planned to display posters in the family waiting rooms of participating ICUs, where required. However, a few ethics committees mandated that research personnel obtain consent from patients or their substitute decision makers for participation. Fifth, although we initially planned to implement the IOS study during two data collection periods of approximately 2 weeks duration each, we recognised early in study implementation that this would not be feasible due to wide variation in the availability and efficiency of ethics and contract approval processes. Moreover, although most ICUs required only one ethics approval to participate, several ICUs especially in the UK and Australia required multiple approvals (eg, central plus other approval). Consequently, we adopted a staggered approach to study implementation. With this approach, we trained local research personnel on the study protocol and data collection procedures after local approvals were obtained and before study activation. Lastly, we underestimated the number of ICUs that we would need to approach to achieve 150 participating ICUs. Personnel from several sites who initially agreed to participate, subsequently withdrew study participation for various reasons. Consequently, ICU recruitment and initiation of approval processes became an iterative process. To achieve our target number of events, we ultimately reduced the minimum number of beds required in participating ICUs from 15 to 9 beds.

Successful study coordination and implementation will require broad collaboration. This study builds on previous work conducted by members of the investigative team within in a Program of Research on Mechanical Ventilation Discontinuation Practices and is being conducted under the auspices of the Canadian Critical Care Trials Group
(CCCTG).$^{29}$ Support for a research programme by the CCCTG signals a commitment to participate actively in the programme's development, refinement, conduct and, ultimately, reporting in the medical literature ${ }^{30}$ Regional site leads (Dr Jones (UK), Dr Villar (Europe), Dr Kapadia (India), Dr Gattas (Australia/New Zealand), Dr Epstein (USA)) will assist with implementing the IOS Study in ICUs in their respective regions, function as local resources, and aid in drafting and revising the manuscript. The members of the organising team in Canada (Dr Burns, Dr Meade, Dr Cook, Dr Dodek, Dr Slutsky and Dr Cook) have strong working relationships with the regional site leads through previous collaborations. We will coordinate study implementation at participating sites using webinars, telephone, facsimile and electronic mail correspondence. To facilitate communication between the study investigators and personnel at participating ICUs, we will launch and up-date a microsite containing the study protocol, data collection forms and study newsletters. The investigative team will own and manage the study database, conduct the planned analyses and draft manuscripts independently from industry partners. Industry partners will be invited to review the study protocol, data collection forms and the study manuscript prior to submission. Industry partners may request and pay for additional analyses.

The IOS study will characterise practices in discontinuing MV and the impact of using various discontinuation strategies on important outcomes. The IOS study is novel in evaluating actual weaning and discontinuation practices outside the structure of randomised trials on an international level and in heterogeneous settings. The IOS study will provide valuable information to the international critical care community, identify practices that warrant further study, inform the design of future trials, and foster collaborations between investigators and regions.

\section{Author affiliations}

${ }^{1}$ Interdepartmental Division of Critical Care, University of Toronto, Toronto, Ontario, Canada

${ }^{2}$ Division of Critical Care Medicine, Department of Medicine, St Michael's Hospital, Toronto, ON, Canada

${ }^{3}$ Li Ka Shing Knowledge Institute, St. Michael's Hospital, Toronto, ON, Canada

${ }^{4}$ Division of Critical Care, St. Joseph's Hospital, Hamilton, Ontario, Canada

${ }^{5}$ Departments of Medicine and Health Research Methods, Evidence and Impact,

McMaster University, Hamilton, ON, Canada

${ }^{6}$ Centre for Health Evaluation and Outcome Sciences and Division of Critical Care Medicine, Division of Critical Care Medicine, St Paul's Hospital and University of British Columbia, Vancouver, BC, Canada

'Department of Critical Care Medicine, Guy's and St. Thomas' NHS Foundation Trust, London, UK

${ }^{8}$ CIBER de Enfermedades Respiratorias, Instituto de Salud Carlos III, Madrid, Spain ${ }^{9}$ Multidisciplinary Organ Dysfunction Evaluation Research Network, Hospital Universitario de Gran Canaria Dr Negrin, Las Palmas de Gran Canaria, Spain

${ }^{10}$ Department of Intensive Care, Hinduja National Hospital, Mumbai, India

${ }^{11}$ Intensive Care Unit, Royal Prince Alfred Hospital, Camperdown, New South Wales, Australia

${ }^{12}$ George Institute for Global Health, Sydney, New South Wales, Australia

${ }^{13}$ Tuft University School of Medicine, Boston, Massachusetts, United States

Acknowledgements We wish to thank the members of the Canadian Critical Care Trials Group for their methodologic advice and content expertise and review of the protocol. 
Collaborators K.E.A. Burns, Deborah J. Cook, Leena Rizvi, Peter Dodek, Maureen 0 . Meade.

Contributors Study design: KB, DC, PD, MM, JV and AS. Study funding: KB, DC, PD and MM. Data collection: KB, LR, AJ, FK, DG and SE. Manuscript preparation: All authors. Manuscript approval: All authors.

Funding This investigator-initiated, peer-reviewed, observational study was funded through a Canadian Institutes of Health Research Operating/Industry-Partnered grant that included contributions from three industry partners (Covidien (Colorado, USA), Fisher \& Paykel (Auckland, New Zealand), and General Electric Healthcare (USA/Finland)) and an International Strategic Opportunities Grant from the Ontario Ministry of Research and Innovation. Additional sources of funding included two personnel awards (Elsie Winnifred Crann Memorial Award, CIHR Clinician Scientist Phase 2 Award) granted to Dr Burns.

Competing interests None declared.

Patient consent for publication Not required.

Ethics approval The protocol for this study was approved by the Ethics Committee of St. Michael's Hospital (\#11-024, Toronto, Canada), the IOS Coordinating Center.

Provenance and peer review Not commissioned; externally peer reviewed.

Open access This is an open access article distributed in accordance with the Creative Commons Attribution Non Commercial (CC BY-NC 4.0) license, which permits others to distribute, remix, adapt, build upon this work non-commercially, and license their derivative works on different terms, provided the original work is properly cited, appropriate credit is given, any changes made indicated, and the use is non-commercial. See: http://creativecommons.org/licenses/by-nc/4.0/.

\section{REFERENCES}

1. Esteban A, Alía I, Ibañez J, et al. Modes of mechanical ventilation and weaning. A national survey of Spanish hospitals. The Spanish lung failure Collaborative group. Chest 1994;106:1188-93.

2. Esteban Aet al. Characteristics and Outcomes in Adult Patients Receiving Mechanical Ventilation<SUBTITLE $>$ A 28-Day International Study</SUBTITLE\&gt. JAMA 2002;287:345-55.

3. Pingleton SK. Complications of acute respiratory failure. Am Rev Respir Dis 1988;137:1463-93.

4. Niederman MS, Ferranti RD, Ziegler A, et al. Respiratory infection complicating long-term tracheostomy : the implication of persistent gram negative tracheobronchial colonization. Chest 1984;85:39-44.

5. Maclntyre NR, Cook DJ, Ely EW Jr, et al. Evidence based guidelines for weaning and discontinuing ventilatory support. A collective Task force facilitated by the American College of chest physicians, the American association for respiratory care and the College of critical care medicine. Chest 2001;120(Supple 6):375S-95.

6. Ely EW, Baker AM, Dunagan DP, et al. Effect on the duration of mechanical ventilation of identifying patients capable of breathing spontaneously. N Engl J Med 1996;335:1864-9.

7. Kollef $\mathrm{MH}$, Shapiro SD, Silver $\mathrm{P}$, et al. A randomized, controlled trial of protocol-directed versus physician-directed weaning from mechanical ventilation. Crit Care Med 1997;25:567-74.

8. Marelich GP, Murin S, Battistella F, et al. Protocol weaning of mechanical ventilation in medical and surgical patients by respiratory care practitioners and nurses. Effect on weaning time and incidence of ventilator associated pneumonia. Chest 2000;118:459-67.

9. Blackwood B, Burns K, Cardwell C, et al. Use of weaning protocols for reducing duration of mechanical ventilation in critically ill adult patients: un updated Cochrane systematic review and meta-analysis. Cochrane Database of Syst Reviews 2014;(Issue 11):CD006904.

10. Esteban A, Alia I, Gordo F, et al. Extubation outcome after spontaneous breathing trials with T-tube or pressure support ventilation. Am J Respir Crit Care Med 1997;156:459-65.
11. Esteban A, Alia I, Tobin MJ, et al. Effect of spontaneous breathing trial duration on outcome of attempts to discontinue mechanical ventilation. Am J Respir Crit Care Med 1999;159:512-8.

12. Perren A, Domenighetti G, Mauri S, et al. Protocol-directed weaning from mechanical ventilation: clinical outcome in patients randomized for a 30-min or 120-min trial with pressure support ventilation. Intensive Care Med 2002;28:1058-63.

13. Burns KEA, Soliman I, Adhikari NKJ, et al. Trials directly comparing alternative spontaneous breathing trial techniques: a systematic review and meta-analysis. Crit Care 2017;21.

14. Brochard L, Rauss A, Benito S, et al. Comparison of three methods of gradual withdrawal from ventilatory support during weaning from mechanical ventilation. Am J Respir Crit Care Med 1994;150:896-903.

15. Esteban A, Frutos F, Tobin MJ, et al. A comparison of four methods of weaning patients from mechanical ventilation. $N$ Engl J Med 1995;332:345-50.

16. Girard TD, Alhazzani W, Kress JP, et al. An official American thoracic Society/American College of chest physicians clinical practice guideline: liberation from mechanical ventilation in critically ill adults. rehabilitation protocols, ventilator liberation protocols, and cuff leak tests. Am J Respir Crit Care Med 2017;195:120-33.

17. Burns KEA, Raptis S, Nisenbaum R, et al. International practice variation in weaning critically ill adults from invasive mechanical ventilation. Ann Am Thorac Soc 2018;15:494-502.

18. Dries DJ, McGonigal MD, Malian MS, et al. Protocol-Driven ventilator weaning reduces use of mechanical ventilation, rate of early Reintubation, and ventilator-associated pneumonia. J Trauma 2004;56:943-52.

19. McLean SE, Jensen LA, Schroeder DG, et al. Improving adherence to a ventilator weaning protocol for critically ill adults: outcomes after an implementation program. Am J Crit Care 2006;15:299-309.

20. Ely EW, Bennett PA, Bowton DL, et al. Large scale implementation of a respiratory Therapist- driven protocol for ventilator weaning. Am J Respir Crit Care Med 1999;159:439-46.

21. Girault C, Breton L, Richard J-C, et al. Mechanical effects of airway Humidification devices in difficult to wean patients*. Crit Care Med 2003;31:1306-11.

22. Streiner DL, Norman GR. Health Measurement Scales - A Practical Guide to Their Development and Use. $4^{\text {th }}$ edition. Oxford, UK: Oxford University Press, 2008.

23. Kleinbaum DG. Applied regression analysis and other multivariable methods. Duxbury Press, 1998: xviii, p 798.

24. Vittinghoff E, McCulloch CE. Relaxing the rule of ten events per variable in logistic and COX regression. Am J Epidemiol 2007;165:710-8.

25. World Medical Association Declaration of Helsinki. Ethical principles for medical research involving human subjects. JAMA 2013;310:2191-4.

26. Burns KEA, Lellouche F, Loisel F, et al. Weaning critically ill adults from invasive mechanical ventilation: a national survey. Can $J$ Anesth/J Can Anesth 2009;56:567-76.

27. Chatburn RL. Classification of ventilator modes: update and proposal for implementation. Respir Care 2007;52:301-23.

28. JV T, Willison DJ, Silver FL, et al. Investigators in the registry of the Canadian stroke network. Impracticability of informed consent in the registry of the Canadian stroke network. $N$ Engl J Med 2004;350:1414-21.

29. Marshall JC, Cook DJ. Investigator-led clinical research consortia: the Canadian critical care Trials Group. Crit Care Med 2009;37(Supp lement):S165-S172.

30. Adams AB, Simonson D. Publications, citations and impact factors of leading Investigators in critical care medicine. Respir Care 2004;49:276-81. 